\title{
Symptomatic Construct Failure after Metastatic Spine Tumor Surgery
}

\author{
Naresh Kumar, Ravish Patel, Jiong Hao Tan, Joshua Song, Naveen Pandita, \\ Dennis Hwee Weng Hey, Leok Lim Lau, Gabriel Liu, Joseph Thambiah, Hee-Kit Wong \\ Department of Orthopaedic Surgery, National University Health System, Singapore
}

\begin{abstract}
Study Design: Retrospective cohort study.
Purpose: To evaluate the incidence and presentation of symptomatic failures (SFs) after metastatic spine tumor surgery (MSTS). To identify the associated risk factors. To categorize SFs based on the management in these patients.

Overview of Literature: Few studies have reported on the incidence $(1.9 \%-16 \%)$ and risk factors of SF after MSTS. It is unclear whether all SFs, occurring in MSTS-patients, result in revision surgery.

Methods: We conducted a retrospective analysis on 288 patients (246 for final analysis) who underwent MSTS between 2005-2015. Data collected were demographics and peri/postoperative clinical and radiological features. Early and late radiological SF were defined as presentation before and after 3 months from index surgery, respectively. Univariate and multivariate models of competing risk regression analysis were designed to determine the risk factors for SF with death as a competing event.

Results: We observed 14 SFs (5.7\%) in 246 patients; 10 (4.1\%) underwent revision surgery. Median survival was 13.4 months. The mean age was 58.8 years (range, 21-87 years); 48.4\% were women. The median time to failure was 5 months (range, 1-60 months). Patients with SF were categorized into three groups: (1) SF when the primary implant was revised ( $n=5,35.7 \%$ ); (2) peri-construct progression of disease requiring extension ( $n=5,35.7 \%$ ); and (3) SFs that did not warrant revision ( $n=4,28.5 \%)$. Four patients (28.5\%) presented with early failure. SF commonly occurred at the implant-bone interface $(9 / 14)$ and all patients had a spinal instability neoplastic score (SINS) $>7$. Thirteen patients $(92.8 \%)$ who developed failure had fixation spanning junctional regions. Multivariate competing risk regression showed that preoperative Eastern Cooperative Oncology Group score was a significant risk factor for implant failure (adjusted sub-hazard ratio, 7.0; 95\% confidence interval, 1.63-30.07; $p<0.0009$ ).

Conclusions: The incidence of SF (5.7\%) was low in patients undergoing MSTS although these patients did not undergo spinal fusion. Preoperative ambulators involved a 7 times higher risk of failure than non-ambulators. Preoperative SINS $>7$ and fixations spanning junctional regions were associated with SF. Majority of construct failures occurred at the implant-bone interface.
\end{abstract}

Keywords: Spine; Neoplasm metastasis; Surgery; Implants, Artificial; Implant failure

\section{Introduction}

Surgical treatment of spinal tumors, such as decompression and fixation with spinal implants, is important in the treatment of metastatic spinal disease (MSD) [1,2]. Spinal fixation aims to treat symptomatic neoplastic instability, allow adequate stabilization of the decompressed segment $[3,4]$ and, in rare cases, bridge the interval between spinal

Received Apr 12, 2020; Revised Jul 1, 2020; Accepted Jul 4, 2020

Corresponding author: Naresh Kumar

Department of Orthopaedic Surgery, National University Health System, Level 11, Tower Block, 1E, Lower Kent Ridge Road, Singapore 119228

Tel: +65-6772-5611, Fax: +65-67780720, E-mail: dosksn@nus.edu.sg 
stabilization and fusion [5]. Successful fusion is vital for long-term stability of the instrumented fixation. However, successful fusion requires a viable host graft bed, favorable biomechanical environment, and adequate nutrient supply $[6,7]$; many of these factors are missing in MSD patients. Advanced age, malnutrition, bone marrow suppression, steroid treatment, chemotherapy (CT), and radiotherapy (RT) are some factors that compromise spinal fusion in MSD patients, making them susceptible to implant/construct failure $[8,9]$. As mentioned, an appropriate bone graft is essential for achieving fusion. However, autograft harvest may lead to donor site morbidity or tumor spread, and allografts are usually avoided owing to the risk of infections and high costs. Hence, due to compromised fusion and limited survival in MSD patients, fixation with or without decompression is the presently accepted treatment, with no attempt being made for fusion [5].

It is postulated that an increase in cancer survival leads to increased incidence of implant failure following metastatic spine tumor surgery (MSTS) $[10,11]$. The reported implant failure rates in a large series of MSD patients ranges between $1.9 \%$ and $16 \%[3,6-9,12,13]$. However, few studies have evaluated the incidence, presentation, and risk factors of implant failures following MSTS [3,12,14]. These studies are heterogeneous with respect to the types of instrumentation and the constructs used [15]. Implant failure can be clinically presented as a spectrum with one end having patients who can be managed non-operatively and the other with patients who show symptomatic failure (SF) and require revision surgery [16].

Therefore, this study was designed with the following objectives: (1) evaluate the incidence and presentation of symptomatic construct failures after MSTS; (2) identify the associated risk factors; and (3) categorize SFs based on the management in these patients.

\section{Materials and Methods}

\section{Subjects}

We present a retrospective study that included patients who underwent spinal fixations with or without decompression for MSD at National University Health System, Singapore in the period 2005-2015. Surgeries were performed by one of five senior consultants. Ethical approval for the study was obtained from the institutional review board (DSRB reference no., 2014/00050). Patients' records were accessed from the hospital's computerized patient support system-2. Informed consent could not be obtained from patients due to the retrospective nature of the study design. Patients with spinal metastasis who were undergoing surgery because of neurological deficits, spinal instability, intractable pain, or any combination of the three were included. Patients aged $<18$ years, who had prior spinal surgery for non-metastatic cause, and with evidence of deep surgical site infections or survival $<30$ days were excluded. Patients were followed up for clinical and radiological assessment at 2 weeks, 6 weeks, 3 months, 6 months, and every 6 months thereafter. However, if the patients needed to consult their surgeon outside these windows, appropriate interim arrangements were made. The endpoint of patients' evaluation was their last followup or death, whichever came earlier.

We collected data regarding patient demographics, tumor type, disease severity, preoperative neurological status, ambulatory status, and preoperative Eastern Cooperative Oncology Group (ECOG) score that represent simple measures of functional status and indications for surgery [17]. Radiological characteristics of metastatic spinal tumors in terms of lytic, sclerotic or mixed pattern, levels involved, and spinal instability neoplastic score (SINS) were determined [18]. Other variables considered for the study included the type of surgical approach (anterior or anterior with posterior for cervical \& posterior only for cervicothoracic/thoracic/thoracolumbar/lumbar/lumbosacral), type of instrumentation, spinal levels involved in instrumentation and decompression, ambulatory status, and postoperative RT.

\section{Definitions}

"Implant failure" was defined as radiologically detectable implant disassembly or breakage with no significant changes in the bone morphology. "Construct failure" was defined as radiologically detectable changes in the construct appearance or integrity (implant or bone). Implant failure/construct failure could possibly develop through one or more of the mechanisms presented in Supplemental Table 1 [16]. Implant failure/construct failure in patients who exhibit pain and/or neurological deficit and/ or deterioration in their mobility from their peak postoperative mobility status were considered "SF" [16]. "Asymptomatic construct failure" was defined as a condition with radiologically detectable changes in the construct appear- 
Table 1. Demographic and clinical data of all patients

\begin{tabular}{|c|c|c|c|}
\hline Characteristic & Category & Total patients & Patients with symptomatic failure \\
\hline Overall & & $246(100.0)$ & $14(5.7)$ \\
\hline Age (yr) & & $58.8 \pm 11.7$ & $57.7 \pm 7.2$ \\
\hline \multirow[t]{2}{*}{ Gender } & Male & $127(51.6)$ & $8(57.1)$ \\
\hline & Female & $119(48.4)$ & $6(42.9)$ \\
\hline \multirow[t]{7}{*}{ Primary tumor } & Lung & $68(27.6)$ & $4(28.6)$ \\
\hline & Breast & $41(16.7)$ & $4(28.6)$ \\
\hline & Renal & $23(9.3)$ & $3(21.4)$ \\
\hline & Prostate & $22(8.9)$ & $1(7.1)$ \\
\hline & Nasopharynx & $10(4.1)$ & $1(7.2)$ \\
\hline & Cervix & $9(3.7)$ & $1(7.2)$ \\
\hline & Others & $73(29.7)$ & 1 (7.1) (thyroid) \\
\hline \multirow[t]{3}{*}{ Type of lesion } & Sclerotic & $29(11.8)$ & $1(7.1)$ \\
\hline & Lytic & $163(66.3)$ & $10(71.4)$ \\
\hline & Mixed & $54(22.0)$ & $3(21.5)$ \\
\hline \multirow[t]{3}{*}{ SINS } & $<7$ (deemed stable) & $26(10.6)$ & 0 \\
\hline & 7-12 (indeterminate) & $178(72.4)$ & $9(64.3)$ \\
\hline & $>12$ (deemed unstable) & $42(17.1)$ & $5(35.7)$ \\
\hline \multirow[t]{2}{*}{ Preoperative ECOG scores } & $0-2$ & $46(18.7)$ & $11(78.5)$ \\
\hline & $3-4$ & $200(81.3)$ & $3(21.4)$ \\
\hline \multirow[t]{7}{*}{ Level of vertebra affected } & Cervical & $22(8.9)$ & $1(7.2)$ \\
\hline & Cervical-thoracic & $18(7.3)$ & $1(7.2)$ \\
\hline & Thoracic & $106(43.1)$ & $5(35.7)$ \\
\hline & Thoracolumbar & $32(13.0)$ & $3(21.4)$ \\
\hline & Lumbar & $52(21.1)$ & $3(21.4)$ \\
\hline & Lumbosacral & $6(2.4)$ & - \\
\hline & Multiple regions involved & $10(4.1)$ & $1(7.1)$ \\
\hline \multirow[t]{6}{*}{ Instrumented levels } & Cervical & $12(4.3)$ & - \\
\hline & Cervical-thoracic & $42(17.1)$ & $3(21.5)$ \\
\hline & Thoracic & $73(29.7)$ & $1(7.1)$ \\
\hline & Thoracolumbar & $83(33.7)$ & $8(57.2)$ \\
\hline & Lumbar & $15(6.1)$ & - \\
\hline & Lumbosacral/thoraco-lumbo-sacral & $20(8.1)$ & $2(14.3)$ \\
\hline \multirow[t]{2}{*}{ Junctional or non-junctional fixations } & Junctional fixation & $145(58.9)$ & $13(92.8)$ \\
\hline & Non-junctional fixation & $101(41.1)$ & $1(7.1)$ \\
\hline \multirow[t]{4}{*}{ Skip instrumentation length } & 0 & $53(21.5)$ & $7(50)$ \\
\hline & 1 & $90(36.6)$ & $4(28.5)$ \\
\hline & 2 & $55(22.4)$ & $1(7.2)$ \\
\hline & $\geq 3$ & $48(19.5)$ & $2(14.3)$ \\
\hline \multirow[t]{3}{*}{ Construct length } & $<6$ & $92(37.4)$ & $6(42.8)$ \\
\hline & $6-9$ & $123(49.3)$ & $7(50.0)$ \\
\hline & $>9$ & 31 (12.6) & $1(7.2)$ \\
\hline \multirow[t]{2}{*}{ MIS or open approach } & MIS & $49(19.9)$ & $4(28.6)$ \\
\hline & Open & $194(78.9)$ & $10(71.4)$ \\
\hline
\end{tabular}

Values are presented as number (\%) or mean \pm standard deviation. A p-value from Likelihood ratio test.

SINS, Spinal Instability Neoplastic Score; ECOG, Eastern Cooperation Oncology Group; MIS, minimally invasive surgery. 
ance or integrity (implant and/or bone), without pain or neurological deficit or deterioration in the mobility from the peak postoperative mobility status [16].

SF can present early or late, with the development of clearly detectable radiological and clinical signs. "Early failure" is defined as presentation of SF within 3 months of the index surgery, while "late failure" is defined by the presentation of SF after 3 months of the index surgery. A time point of 3 months was rationally selected to distinguish early failures from late failures. Most patients patients were subjected to RT and/or CT about 2-3 weeks after MSTS and re-calcification of the affected vertebra indicating local disease control, takes about 1-3 months $[19,20]$. Therefore, the load sharing capacity of the affected vertebrae can be reasonably assumed to increase from 3 months after the index surgery.

Implant/construct failures were evaluated radiologically and documented as screw failure (ploughing, loosening, cut-out, pull-out, breakage), cage failure (subsidence/tilting/translation or breakage), rod breakage, or change $>5^{\circ}$ in the overall sagittal angulation [16]. Implant failure was clinically evaluated for new-onset deformity or neurological deficit with or without pain and/or any deterioration in the ambulatory state. Pain was assessed using the visual analog scale preoperatively and postoperatively. They were stratified into asymptomatic or SFs as per their clinical presentation.

Statistical tests were conducted using STATA ver. 11.0 (Stata Corp., College Station, TX, USA) and R software ver. 3.6.3 (The R Foundation for Statistical Computing, Vienna, Austria; https://www.r-project.org/). Univariate and multivariate models of competing risk regression analysis were designed to determine the risk factors for SF with death as a competing event. The "cmprsk" package of R software ver. 3.6.3 (The R Foundation for Statistical Computing) was utilized to generate the cumulative frequency curve that compared the incidence of SF and death. A $p$-value $<0.05$ was considered to indicate statistical significance.

\section{Results}

Total 288 patients underwent MSTS between 2005 and 2015. Forty-two patients were excluded because they died within 30 days of the procedure or their MSTS did not involve instrumentation; thus, the final analysis included 246 patients. SF was observed in 5.7\% (14/246) of the sub- jects, and 71\% (10/14) underwent revision. All SFs were construct failures; however, no SF was a true implant failure. Majority $64.3 \%$ (9/14) of SFs occurred at the implantbone interface. Forty-one 16\% (41/246) patients showed radiological evidence of implant/construct failure without clinical symptoms at the end of the study.

The mean age at the time of surgery was 58.8 years (range, 21-87 years). The study population included 127 men (51.6\%) and 119 women (48.4\%). The common tumor types were lung cancer $(27.6 \%, \mathrm{n}=68)$, breast cancer $(16.7 \%$, $\mathrm{n}=41)$, hematological cancer $(12.9 \%, \mathrm{n}=30)$, hepatocellular/gastrointestinal cancer $(10.9 \%, n=27)$, kidney cancer $(9.3 \%, \mathrm{n}=23)$, and prostate cancer $(8.9 \%, \mathrm{n}=22)$. Majority (66.3\%) of the lesions were lytic. Instrumented region, levels of instrumentation, and number of skipped vertebrae are shown in Table 1. The median survival duration was 13.4 months (range, 1-127 months). The median survival in patients with SF was 21 months (range, 4-69 months). The mean interval between index surgery and clinical presentation of SF was $12.8 \pm 17.7$ months. The median time to failure was 5 months (range, 1-60 months). Presentation of SF in patients involved intractable pain, 78.5\% (11/14); new-onset neurological deficit, 50\% (7/14); implant prominence, $14.2 \%(2 / 14)$, and secondary wound dehiscence, $7.1 \%(1 / 14)$. Nine failures (64.3\%) had SINS 7-12 and five failures (35.7\%) had SINS $>12$; however, no failures had SINS $<7$. In patients with SF, 13/14 (92.8\%) had fixations spanning a junctional region. Forty-nine patients (19.9\%) were operated with MIS, while 194 (78.9\%) were managed using open surgery. On subgroup analysis for the surgery type in SF patients, MIS was performed for four patients (28.6), while open surgery was performed for 10 patients (71.4\%). No significant correlation was found between the surgery type and SF.

Patients with SF were categorized into the following three groups: (1) patients who underwent revision $(n=5$, $35.7 \%)$; (2) peri-construct or local tumor progression that required construct extension ( $\mathrm{n}=5,35.7 \%)$; and (3) the patients who did not undergo revision surgery due to prevalent comorbidities, surgical unfeasibility, or refusal of surgical intervention $(n=4,28.5 \%)$. In the third group, two patients (case 7 and 9) were bed-bound and terminally ill, one (case 6) refused to undergo surgery owing to fear of imminent terminal event, and one (case 8) had an inoperable tumor (Supplemental Table 2). Hence, only $71 \%$ patients (10/14) with SF underwent revision surgery in our study. 
Early failures (within 3 months of the index surgery) were observed in four patients (28.5\%). Cases 3 and 7 presented with progressive construct kyphosis and screw ploughing (Table 2, Supplemental Table 2). Patient 8 had cage subsidence and screw cutout, while patient 2 had screw pull-out leading to implant prominence and wound dehiscence (Table 2, Supplemental Table 2). All these patients had an ECOG score between 0 and 2 .

Late failures ( $>3$ months from the index surgery) were observed in 10 patients (71.5\%). Case 1 had screw cut-out with signs of cord compression (Fig. 1). Patient 5 presented with reduced lumbar lordosis and decreased anterior

Table 2. Frequency of the radiological failure mechanisms in patients with symptomatic failures

\begin{tabular}{lc}
\hline Failure mechanism & No. of patients \\
\hline Screw ploughing & 2 \\
\hline Screw loosening & 0 \\
\hline Screw pull-out & 5 \\
\hline Screw cut-out & 3 \\
\hline Screw breakage & 0 \\
\hline Cage subsidence & 3 \\
\hline Cage displacement & 0 \\
\hline Cage breakage & 0 \\
\hline Rod breakage & 0 \\
\hline Angular deformity & 5 \\
\hline Increase in kyphosis & 3 \\
\hline Decrease lordosis & 2 \\
\hline Peri-construct disease progression & 5 \\
\hline Total & 23 \\
\hline
\end{tabular}

column height with screw pull-out (Fig. 2). Of these failures, five patients were secondary to peri-construct disease progression or peri-construct failures (Fig. 3, Table 2, Supplemental Table 2). Rod or screw breakage was not observed in any patient.

Univariate and multivariate competing risk regression model showed that preoperative ambulatory status (ECOG $\leq 2$ ) is a significant risk factor for SF with mortality as a competing event. Before the surgery, 11 patients (78.5\%) with SF were ambulatory, while 3 (21.4\%) were non-ambulatory. Ambulatory patients had a 7 times higher risk of developing implant failure (Table 3). Moreover, SINS $>7$ was a risk factor because all patients with SF had SINS $>7$ $(p<0.005)$. Similarly, SINS score $>12$ was also found to be significant. The subgroup analysis between the above two groups revealed that the rate of SF was higher 11.9\% (5/42) in those with SINS score $>12$ as compared to that in those with a SINS score 7-12, 5\% (9/178). This difference was statistically significant $(p<0.05)$ (Table 3$)$. Fixations spanning junctional areas were another risk factor $(p<0.005)$ because $93 \%$ (13/14) of patients with SF had fixations spanning junctional areas.

The "cmprsk" package of R software (The R Foundation for Statistical Computing) was used to plot the probability of SF and probability of death without developing SF. The cumulative frequency curves depict that the probability of death without developing implant failure is $70 \%$, and the probability of SF was $5 \%$ at the end of 2 years of follow-up (Fig. 4). However, the projection of the graph beyond the study period shows that the SF peaks at $10 \%$, while the probability of death (80\%) always remains significantly higher than the probability of SF. This finding favors our
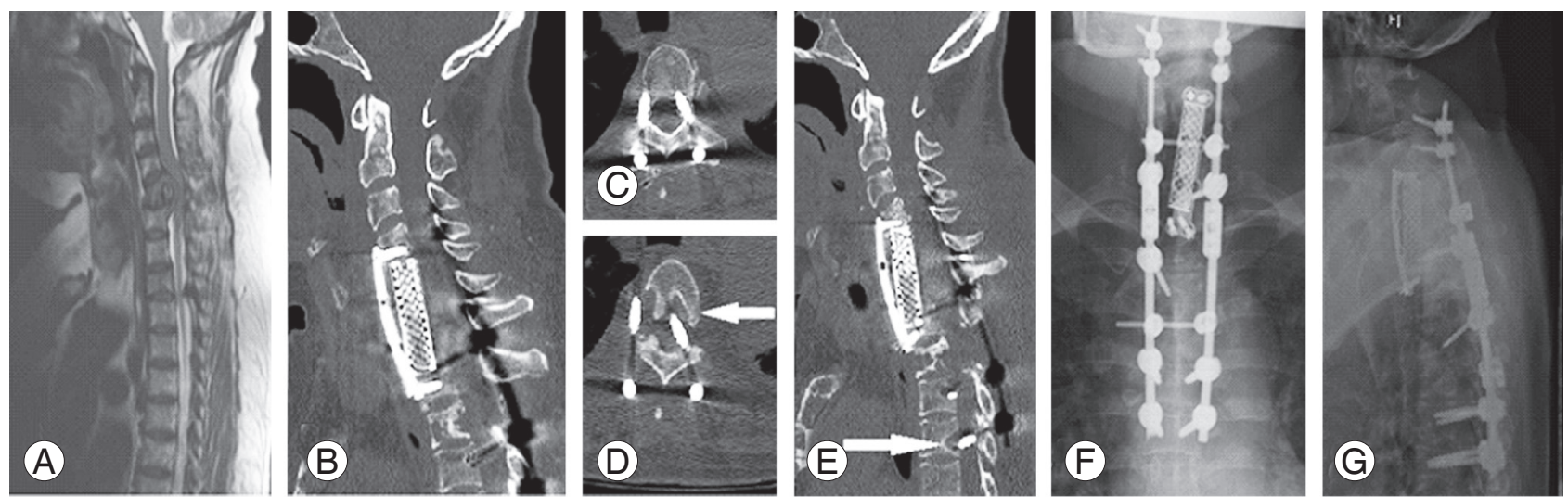

Fig. 1. Case 1: (A) 49-year-old female patient with spinal metastases from breast to C5-C6, T1-T2. (B, C) C3-T4 posterior stabilization, C5-C7 corpectomy and posterior decompression of T2. (D, E) T4 cord compression secondary to screw cut-out (arrow). (F, G) Reposition of T4 screws and extension of instrumentation to T6. 
observation that longer life expectancy in MSTS patients was not associated with SF. We also found that patient age at the time of surgery was not a risk factor for the development of SF (Table 3, Fig. 4).

\section{Discussion}

The current study was designed to evaluate the rate of SF in MSD and identify the associated risk factors. SF is a significant postoperative event that affects the life quality of patients who undergo MSTS. Hence, it is vital to analyze the SF risk in these patients. In our series, the SF rate was $5.7 \%(14 / 246)$, and the revision rate was $4.1 \%(10 / 246)$. Of the 14 SFs, 5 (35.7\%) were attributable to peri-construct disease progression, secondary to failed RT/CT, or appearance of newer lesions or peri-construct failures.
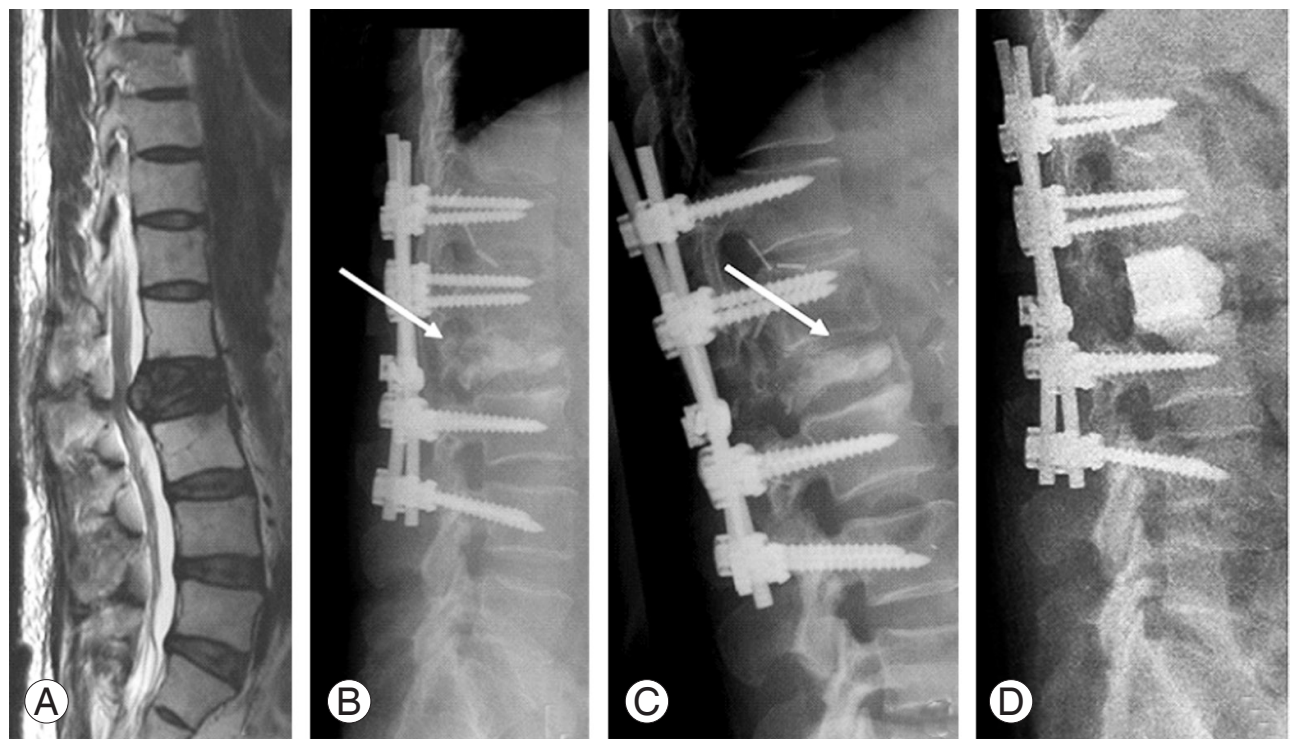

Fig. 2. Case 5: (A) 56-year-old male patient with spinal metastases from kidney to L2. (B) T12-L4 posterior stabilization (arrow). (C) Reduced anterior column height and reduced lordosis (arrow). (D) Revision anterior corpectomy and reconstruction of L2 done with placement of cage filled with cement.
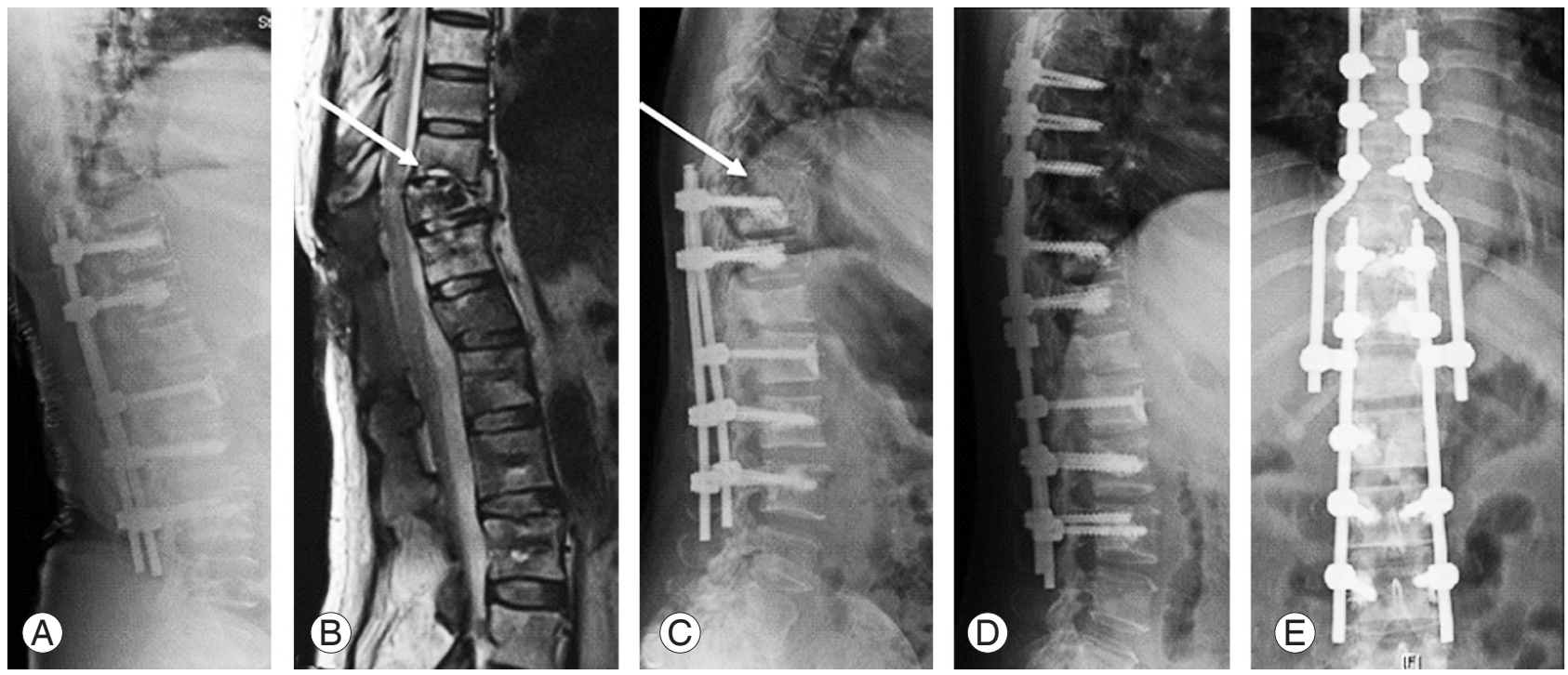

Fig. 3. Case 4: 54-year-old female patient with spinal metastases from nasopharynx to T11, T12. (A, B) Posterior stabilization from T10-L3 and decompression of T11-T12 (arrow). (C) Fracture of the end plates in T9 and T10 with screw cut-out and screw pull-out (arrow). (D, E) Extension of posterior instrumentation from T6-L3; decompression of $\mathrm{T} 9$ and $\mathrm{T} 11$. 
These five patients were included as we believe that periconstruct progression of tumors will compromise the existing constructs, requiring extension or revision of the implants. Resistance to RT/CT is an important factor for peri-construct disease progression apart from de-novo seeding at the periphery of the construct. Hence, failure of RT/CT may lead to an imminent SF. Amankulor et al. [12] noticed that $33 \%$ (3/9) of the failures had peri-construct disease progression or peri-construct failures requiring extension or revision. Peri-construct failures are a definite entity that form a subset of SF.

The median and mean survival times for SF patients in our study were 13.4 months and 21 months, respectively. The reported SF rates after MSTS in the available literature range between $1.9 \%$ and $16 \%[3,6-9,12,13]$. Bellato et al. [3] observed that only $8 \%(9 / 105)$ of the patients undergoing posterior instrumented fusion and decompression, showed radiological evidence of SF with median time to failure being 9.5 months, and no patients required revision surgery. However, Amankulor et al. [12] observed that $2.8 \%(9 / 318)$ of patients undergoing "separation surgery" required revision due to SF with a median survival duration of 13 months. Similarly, Quraishi et al. [21] reported a reoperation rate of $3.2 \%$ in 289 patients over an 8-year study period; Vrionis and Small [7] reported a rate of $4.2 \%$ (3/71) for SF; and Pedreira et al. [13] observed $1.9 \%(3 / 159)$ failures in patients undergoing MSTS. The marginally higher SF rate in our study as compared to that in previous studies may be attributable to the inclusion of

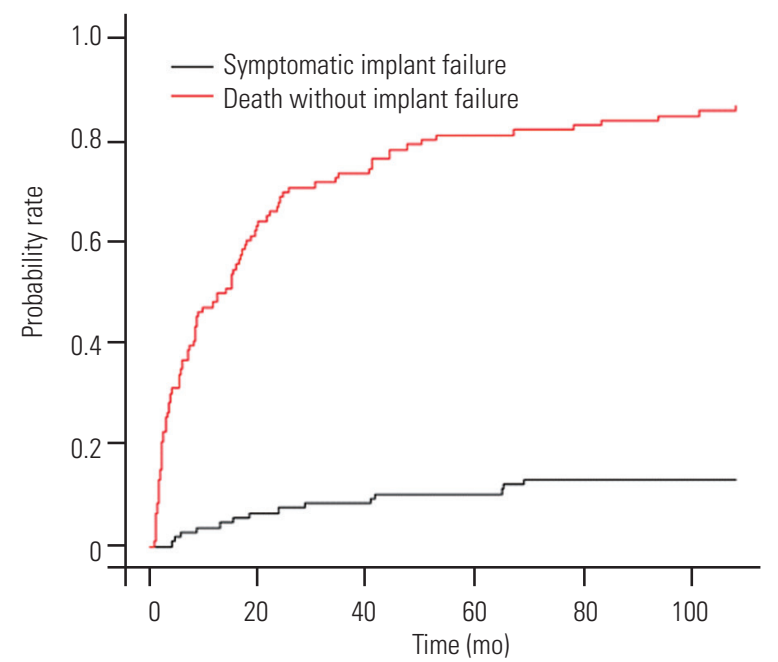

Fig. 4. Graph showing cumulative incidence of patients with implant/construct failure and death without implant failure.
Table 3. Multivariate competing risk regression analysis

\begin{tabular}{lcc} 
Variable & Adjusted SHR $(95 \% \mathrm{CI})$ & $p$-value \\
\hline Age & $0.975(0.906-1.049)$ & 0.504 \\
Sex & & \\
$\quad$ Male & 1.0 & - \\
$\quad$ Female & $3.512(0.653-18.885)$ & 0.143
\end{tabular}

Skipped region

\begin{tabular}{|c|c|c|}
\hline 0 & 1.0 & - \\
\hline 1 & $0.157(0.043-0.563)$ & 0.17 \\
\hline 2 & $0.048(0.001-1.441)$ & 0.08 \\
\hline$\geq 3$ & $0.209(0.020-2.154)$ & 0.189 \\
\hline \multicolumn{3}{|l|}{ Metastatic region } \\
\hline Cervical & 1.0 & - \\
\hline Cervicothoracic & $1.560(0.026-9.270)$ & 0.831 \\
\hline Thoracic & $2.159(0.045-10.244)$ & 0.696 \\
\hline Thoracolumbar & $0.462(0.003-5.432)$ & 0.751 \\
\hline Lumbar & $6.330(0.075-5.331)$ & 0.415 \\
\hline Lumbosacral & $2.554(0.050-1.279)$ & 0.639 \\
\hline \multicolumn{3}{|l|}{ Primary tumor } \\
\hline Lung & 1.0 & - \\
\hline Breast & $0.269(0.040-1.7903)$ & 0.175 \\
\hline Renal & $1.827(0.122-27.253)$ & 0.662 \\
\hline Prostate & $1.646(0.169-16.045)$ & 0.668 \\
\hline Others ${ }^{a}$ & $0.059(0.002-1.435)$ & 0.082 \\
\hline SINS & $1.302(0.758-2.237)$ & 0.338 \\
\hline \multicolumn{3}{|l|}{ Lesion type } \\
\hline Sclerotic & 1.0 & - \\
\hline Lytic & $0.500(0.040-6.110)$ & 0.588 \\
\hline Mixed & $0.438(0.076-2.520)$ & 0.355 \\
\hline \multicolumn{3}{|l|}{ Instrumented region } \\
\hline Cervicothoracic & 1.0 & - \\
\hline Thoracic & $0.559(0.024-12.986)$ & 0.718 \\
\hline Thoracolumbar & $2.688(0.176-40.955)$ & 0.477 \\
\hline Lumbar \& lumbosacral & $0.906(0.041-19.567)$ & 0.95 \\
\hline Construct length & 1.066 (0.816-1.392) & 0.638 \\
\hline \multicolumn{3}{|l|}{ ECOG score } \\
\hline 3-4 (non-ambulatory) & 1.0 & - \\
\hline 0-2 (ambulatory) & 7.021 (1.639-30.078) & 0.0009 \\
\hline
\end{tabular}

Bold type is considered as statistically significant. Competing risk regression (multivariate model) with symptomatic failure as event of interest and death as the competing event.

SHR, sub-hazard ratio; Cl, confidence interval; SINS, Spinal Instability Neoplastic Score; ECOG, Eastern Cooperative Oncology Group.

a) Include hematological malignancies $(n=30)$, hepatocellular carcinoma/gastrointestinal tumors $(n=27)$, ovarian $(n=7)$, cervical $(n=9)$, thyroid $(n=7)$, nasopharyngeal $(n=10)$, and others $(n=2)$. 
patients with peri-construct tumor progression or periconstruct failure $[5,12,13,21]$. This is supported by Park et al. [5] in their recent series on 136 patients of MSTS with instrumentation and no fusion; at the 16-month followup, the reported SF rate was 2.2\%; however, they noticed that $4.4 \%$ of their cohort had asymptomatic construct failures.

The implant failure rates following degenerative spinal surgery and degenerative deformity correction are reported to be $4 \%-8 \%$ [22] and $4 \%-44 \%[23,24]$, respectively. In contradiction, the SF rate after MSTS was much lower despite spinal fusion not being attempted or achieved. As observed in our series, the cumulative frequency of death without SF is superior to the SF risk (Fig. 4). Thus, fusion may not be necessary in most MSTS patients. The current literature supports the present philosophy of not aiming for fusion after MSTS [5,12].

In our study, the median interval between the index surgery and detection of failure was 5 months (range, 1-60 months). Early failure was observed in $28.5 \%(n=4)$, while late failure was seen in $71.5 \%(n=10)$, showing that longer survival is associated with SF after MSTS in our series. However, this correlation was not statistically significant (Fig. 4). It is noteworthy that Bellato et al. [3] noticed a larger median time interval to failure of 9.5 months that was higher than the survival in $88 \%$ of their SF patients. This would imply that in their series, out of 13 patients who survived for $\geq 9.5$ months, four were likely to develop hardware failure (30\%). Similarly, Amankulor et al. [12] noticed that $33 \%$ (3/9) of failures occurred within 3 months of index surgery; however, the median time to failure was 23 months. Majority of their patients with a longer survival had late implant failures. Shorter SF duration in our series could be attributed to multifactorial reasons affecting the bone quality and implant purchase.

Longer constructs, chest wall resections, preoperative radiation, and lack of anterior structural support/replacement were considered as causes of SF in previous studies $[3,6,12,13]$. However, in our study, preoperative ECOG scores, SINS score, demographic parameters, construct length, instrumented region, primary tumor type, lesion type, and pre- or postoperative RT were significant risk factors for SF. The univariate and multivariate competing risk regression models showed only preoperative ambulatory status $(\mathrm{ECOG} \leq 2)$ as a significant risk factor for SF with mortality as a competing event $(p=0.0009)$ (Table 3 ). Therefore, it can be concluded that preoperative ambula- tory status as a risk factor for SF in MSD patients is an important consideration. There is a 7 -fold higher risk of developing SF in ambulatory patients than in non-ambulators. Highly active and mobile patients exhibit undue cyclical loading on constructs and may be predisposed to SF [16]. Patients who are neurologically intact, with good local tumor control with RT/CT, and those who are independent or community ambulators are at increased risk of developing late SF because their implants are subjected to cyclical loading during ambulation. The higher frequency of loading and unloading of implants increases the biomechanical stress on the constructs. This is particularly true in patients undergoing limited surgery where the tumor mass is resected intra-lesionally with limited or no reconstruction. As observed in our study, all the patients in the early failure group and $62.5 \%(5 / 8)$ of them in the late failure group had preoperative ECOG score of 0-2.

Amankulor et al. [12] observed that constructs spanning $>6$ levels and chest wall resections were significant risk factors predicting SF following separation surgery via a posterior approach. Placantonakis et al. [6] observed that chest wall resections and spinal systems other than pedicle screw systems are risk factors for implant failure in patients with cervicothoracic metastases. Our study did not contain patients with chest wall resection because our center does not consider a major chest wall procedure to be performed simultaneously with MSTS. If the chest lesion requires addressing, it would be dealt with RT/CT or video-assisted thoracoscopic surgery. Our center reserves such an aggressive procedure of chest wall resection for a solitary primary spinal tumor only.

Common mechanisms previously reported for instrumentation failure in tumor patients were rod breakage, pedicle screw pull-out, and cage migration $[3,12,13]$. We observed that screw pull-out (5/14), peri-construct failure (5/14), cage subsidence (3/14), and an increase in kyphosis (3/14) were common mechanisms in SF (Table 2 ). Our study differs from others in that there were no rod or screw breakages and most SFs occurred at the implantbone interface. This discrepancy may be multifactorial. The primary tumor type, construct selection, construct length, osteoporosis severity, the type and the timing of adjuvant therapy may influence the SF mode. In our study, it was observed that constructs have a tendency to collapse in kyphosis as most tumors are located in thoracic and thoracolumbar regions where the axis of loading is through the anterior column [25]. An increase in kyphosis 
or decrease in lordosis were observed not only in patients with posterior only constructs with fixation but in patients who underwent separation surgery as well. The osteoporotic vertebrae near the affected vertebrae is predisposed to screw ploughing on repetitive loading [26].

It is noteworthy that we observed that preoperative SINS and fixations spanning junctional regions were important factors correlating with SF, although they were not statistically significant after multivariate regression analysis ( $p=0.338, p=0.718, p=0.477$, and $p=0.95$, respectively) (Table 3 ). SINS is a highly reliable, reproducible, and validated assessment tool for the assessment of spinal instability [27]. None of the patients undergoing MSTS with SINS $<7$ developed SF. SINS score 7-12 and SINS score $>12$ both were significant for SF. The subgroup analysis showed that SINS score $>12$ was statistically the most significant to predict SF $(p<0.05)$. This implies that SF may be more common in patients with greater preoperative instability. Out of 14 failures, 13 occurred with fixations involving junctional regions. We found that $57 \%$ $(8 / 14), 21.5 \%(3 / 14)$, and $14 \%(2 / 14)$ of failures were in the thoracolumbar fixations, cervicothoracic fixations, and lumbosacral fixations respectively. Junctional regions are highly mobile segments, thereby rendering their fixations susceptible to repetitive loading leading to SF [27]. Repetitive cyclical loading in ambulatory patients plays an important role in SF after MSTS [16].

With our study and various others reporting different risk factors for SF, it is difficult to reach a consensus on its cause. Our study supports good preoperative ambulatory status, fixations spanning junctional regions, and spinal instability (SINS $>7$ ) as factors predisposing SF.

There are certain limitations of our study because it is a retrospective study spanning over a 10 -year period. Surgical techniques and planning have evolved over the decade resulting in variability in construct selection, surgical planning, and techniques. Further variations were introduced through the individual variation in the decision process of the five different consultants. These variations may affect the surgical outcomes and SF rates. The variability in speed of pain aggravation and functional deterioration could not be commented upon because of the retrospective nature of our study. We analyzed several patient and surgical factors that can contribute to SF; however, additional factors like implant density and autofusion rates were not evaluated as they were beyond the scope of our study. However, given the large number of patients, we believe our study to be representative of the incidence and trends of SF after MSTS.

\section{Conclusions}

The incidence of SF (5.7\%) was low in patients undergoing MSTS despite not undergoing spinal fusion. Our study highlights that preoperative ambulators had a 7 times higher risk of failure than non-ambulators; a preoperative SINS score $>7$ and fixations spanning junctional regions are associated with SF. These factors need to be considered when planning an instrumented stabilization for MSD patients.

\section{Conflict of Interest}

No potential conflict of interest relevant to this article was reported.

\section{Acknowledgments}

We thank Dr. Aye Sandar Zaw for the preparation of "Supplemental Table 2" and statistical analysis. We thank Ms. Sirisha Madhu \& Mr. Sridharan Alathur Ramakrishnan for editing the final document.

\section{Supplementary Materials}

Supplementary materials can be available from https://doi. org/10.31616/asj.2020.0166. Supplemental Table 1. Mechanisms of failure. Supplemental Table 2. Demographics, preoperative and operative characteristics of individual patients exhibiting symptomatic implant failure.

\section{References}

1. Bilsky MH, Laufer I, Burch S. Shifting paradigms in the treatment of metastatic spine disease. Spine (Phila Pa 1976) 2009;34(22 Suppl):S101-7.

2. Patchell RA, Tibbs PA, Regine WF, et al. Direct decompressive surgical resection in the treatment of spinal cord compression caused by metastatic cancer: a randomised trial. Lancet 2005;366:643-8.

3. Bellato RT, Teixeira WG, Torelli AG, Cristante AF, de Barros TE, de Camargo OP. Late failure of posterior fixation without bone fusion for vertebral metastases. Acta Ortop Bras 2015;23:303-6. 
4. Fourney DR, Gokaslan ZL. Spinal instability and deformity due to neoplastic conditions. Neurosurg Focus 2003;14:e8.

5. Park SJ, Lee KH, Lee CS, et al. Instrumented surgical treatment for metastatic spinal tumors: is fusion necessary? J Neurosurg Spine 2019:1-9.

6. Placantonakis DG, Laufer I, Wang JC, Beria JS, Boland P, Bilsky M. Posterior stabilization strategies following resection of cervicothoracic junction tumors: review of 90 consecutive cases. J Neurosurg Spine 2008;9:111-9.

7. Vrionis FD, Small J. Surgical management of metastatic spinal neoplasms. Neurosurg Focus 2003;15:E12.

8. Akeyson EW, McCutcheon IE. Single-stage posterior vertebrectomy and replacement combined with posterior instrumentation for spinal metastasis. J Neurosurg 1996;85:211-20.

9. Wang JC, Boland P, Mitra N, et al. Single-stage posterolateral transpedicular approach for resection of epidural metastatic spine tumors involving the vertebral body with circumferential reconstruction: results in 140 patients: invited submission from the Joint Section Meeting on Disorders of the Spine and Peripheral Nerves, March 2004. J Neurosurg Spine 2004;1:287-98.

10. Jacobs WB, Perrin RG. Evaluation and treatment of spinal metastases: an overview. Neurosurg Focus 2001;11:e10.

11. Kumar N, Malhotra R, Zaw AS, et al. Evolution in treatment strategy for metastatic spine disease: presently evolving modalities. Eur J Surg Oncol 2017;43:1784-801.

12. Amankulor NM, Xu R, Iorgulescu JB, et al. The incidence and patterns of hardware failure after separation surgery in patients with spinal metastatic tumors. Spine J 2014;14:1850-9.

13. Pedreira R, Abu-Bonsrah N, Karim Ahmed A, et al. Hardware failure in patients with metastatic cancer to the spine. J Clin Neurosci 2017;45:166-71.

14. Park SB, Kim KJ, Han S, Oh S, Kim CH, Chung CK. Instrumentation failure after partial corpectomy with instrumentation of a metastatic spine. J Korean Neurosurg Soc 2018;61:415-23.

15. Mesfin A, Sciubba DM, Dea N, et al. Changing the adverse event profile in metastatic spine surgery: an evidence-based approach to target wound complications and instrumentation failure. Spine (Phila $\mathrm{Pa}$
1976) 2016;41 Suppl 20:S262-70.

16. Kumar N, Patel R, Wadhwa AC, et al. Basic concepts in metal work failure after metastatic spine tumour surgery. Eur Spine J 2018;27:806-14.

17. Oken MM, Creech RH, Tormey DC, et al. Toxicity and response criteria of the Eastern Cooperative Oncology Group. Am J Clin Oncol 1982;5:649-55.

18. Fisher CG, DiPaola CP, Ryken TC, et al. A novel classification system for spinal instability in neoplastic disease: an evidence-based approach and expert consensus from the Spine Oncology Study Group. Spine (Phila Pa 1976) 2010;35:E1221-9.

19. Greenburg EJ, Chu FC, Dwyer AJ, Ziminski EM, Dimich AB, Laughlin JS. Effects of radiation therapy on bone lesions as measured by $47 \mathrm{Ca}$ and $85 \mathrm{Sr}$ local kinetics. J Nucl Med 1972;13:747-51.

20. Libshitz HI, Hortobagyi GN. Radiographic evaluation of therapeutic response in bony metastases of breast cancer. Skeletal Radiol 1981;7:159-65.

21. Quraishi NA, Rajabian A, Spencer A, et al. Reoperation rates in the surgical treatment of spinal metastases. Spine J 2015;15(3 Suppl):S37-43.

22. Kim KT, Lee SH, Lee YH, Bae SC, Suk KS. Clinical outcomes of 3 fusion methods through the posterior approach in the lumbar spine. Spine (Phila Pa 1976) 2006;31:1351-8.

23. Mok JM, Cloyd JM, Bradford DS, et al. Reoperation after primary fusion for adult spinal deformity: rate, reason, and timing. Spine (Phila Pa 1976) 2009;34:832-9.

24. Pichelmann MA, Lenke LG, Bridwell KH, Good CR, O'Leary PT, Sides BA. Revision rates following primary adult spinal deformity surgery: six hundred forty-three consecutive patients followed-up to twenty-two years postoperative. Spine (Phila Pa 1976) 2010;35:219-26.

25. Polly DW Jr, Klemme WR, Cunningham BW, Burnette JB, Haggerty CJ, Oda I. The biomechanical significance of anterior column support in a simulated single-level spinal fusion. J Spinal Disord 2000;13:58-62.

26. Drake MT. Osteoporosis and cancer. Curr Osteoporos Rep 2013;11:163-70.

27. Fisher CG, Schouten R, Versteeg AL, et al. Reliability of the Spinal Instability Neoplastic Score (SINS) among radiation oncologists: an assessment of instability secondary to spinal metastases. Radiat Oncol 2014;9:69. 